ARTICLE

\title{
Electrical switching between exciton dissociation to exciton funneling in $\mathrm{MoSe}_{2} / \mathrm{WS}_{2}$ heterostructure
}

\author{
Yuze Meng ${ }^{1,2,6}$, Tianmeng Wang ${ }^{1,6}$, Chenhao Jin (10 ${ }^{3,6}$, Zhipeng Li (10 1, Shengnan Miao ${ }^{1}$, Zhen Lian', \\ Takashi Taniguchi ${ }^{4}$, Kenji Watanabe (ib) ${ }^{4}$, Fengqi Song (i) ${ }^{2 凶} \&$ Su-Fei Shi ${ }^{1}{ }^{1,5}{ }^{凶}$
}

The heterostructure of monolayer transition metal dichalcogenides (TMDCs) provides a unique platform to manipulate exciton dynamics. The ultrafast carrier transfer across the van der Waals interface of the TMDC hetero-bilayer can efficiently separate electrons and holes in the intralayer excitons with a type II alignment, but it will funnel excitons into one layer with a type I alignment. In this work, we demonstrate the reversible switch from exciton dissociation to exciton funneling in a $\mathrm{MoSe}_{2} / \mathrm{WS}_{2}$ heterostructure, which manifests itself as the photoluminescence $(\mathrm{PL})$ quenching to $\mathrm{PL}$ enhancement transition. This transition was realized through effectively controlling the quantum capacitance of both $\mathrm{MoSe}_{2}$ and $\mathrm{WS}_{2}$ layers with gating. PL excitation spectroscopy study unveils that PL enhancement arises from the blockage of the optically excited electron transfer from $\mathrm{MoSe}_{2}$ to $\mathrm{WS}_{2}$. Our work demonstrates electrical control of photoexcited carrier transfer across the van der Waals interface, the understanding of which promises applications in quantum optoelectronics.

\footnotetext{
${ }^{1}$ Department of Chemical and Biological Engineering, Rensselaer Polytechnic Institute, Troy, NY 12180, USA. ${ }^{2}$ National Laboratory of Solid State Microstructures, Collaborative Innovation Center of Advanced Microstructures, and School of Physics, Nanjing University, 210093 Nanjing, P. R. China. ${ }^{3}$ Kavli Institute at Cornell for Nanoscale Science, Cornell University, Ithaca, NY 14853, USA. ${ }^{4}$ National Institute for Materials Science, 1-1 Namiki, Tsukuba 3050044, Japan. ${ }^{5}$ Department of Electrical Computer \& Systems Engineering, Rensselaer Polytechnic Institute, Troy, NY 12180, USA. ${ }^{6}$ These authors

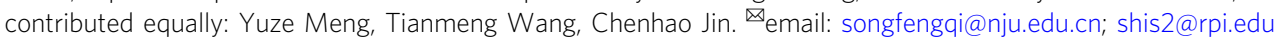


$\mathrm{T}$ wo-dimensional (2D) semiconductors are promising candidates for light-harvesting and optoelectronic applications ${ }^{1-5}$ due to their strong light-matter interaction from excitonic responses ${ }^{6-13}$. Their atomically thin nature further enables engineering exciton dynamics and energy relaxation pathways through ultrafast carrier transfer across $2 \mathrm{D}$ van der Waals (vdW) interfaces ${ }^{14-21}$. In particular, a vdW heterostructure can, respectively, dissociate electrons and holes into separate layers or funnel excitons to one layer with a type II or type I band alignment ${ }^{15,21-28}$. It is highly desirable to achieve both functions in a single device in an electrically reconfigurable way. However, to the best of our knowledge, this has not been demonstrated yet. Here we demonstrate reversible electrical switching between exciton dissociation and funneling in a $\mathrm{MoSe}_{2} / \mathrm{WS}_{2}$ heterostructure device. We show that the electron transfer from $\mathrm{MoSe}_{2}$ to $\mathrm{WS}_{2}$ can be blocked by efficient gating of the $\mathrm{LaF}_{3}$ substrate, leading to a transition between photoluminescence (PL) quenching to PL enhancement for the $\mathrm{MoSe}_{2}$ A exciton emission. The ability to electrically control interlayer charge transfer pathways ushers in application concepts, such as light switch and energy steering.

\section{Results}

Charge transfer in the $\mathrm{MoSe}_{2} / \mathrm{WS}_{2}$ heterostructure. We construct the $\mathrm{MoSe}_{2} / \mathrm{WS}_{2}$ heterostructure on the $\mathrm{LaF}_{3}$ substrate through a layer-by-layer dry transfer technique ${ }^{29}$, and the heterostructure is also capped by a thin layer of hexagonal boron nitride (BN) on the top. A typical $\mathrm{MoSe}_{2} / \mathrm{WS}_{2}$ heterostructure on the $\mathrm{LaF}_{3}$ substrate is shown in Fig. 1a. The overlapped region of the monolayer $\mathrm{MoSe}_{2}$ and $\mathrm{WS}_{2}$ forms the $\mathrm{MoSe}_{2} / \mathrm{WS}_{2}$ heterojunction. We use few-layer flakes of graphene to contact both the monolayer $\mathrm{MoSe}_{2}$ and $\mathrm{WS}_{2}$, and a schematic of the device is shown in Fig. 1b. The heterostructure can be gated through the $\mathrm{LaF}_{3}$ substrate as the back gate, which provides efficient control of doping through the double layer ${ }^{30}$, as schematically shown in Fig. 1b. Typical PL spectra for different regions of the device are shown in Fig. 1c, with the continuous wave (CW) laser excitation centered at $2.331 \mathrm{eV}$ and a power of $100 \mu \mathrm{W}$. Without applying any gate voltage, the PL from the $\mathrm{MoSe}_{2} / \mathrm{WS}_{2}$ heterojunction (red line in Fig. 1c) exhibits quenching of both the PL at the $\mathrm{WS}_{2} \mathrm{~A}$ exciton resonance $(\sim 1.979 \mathrm{eV})$ and $\mathrm{MoSe}_{2} \mathrm{~A}$ exciton resonance $(\sim 1.548 \mathrm{eV})$, compared with that of the monolayer $\mathrm{WS}_{2}$ (blue line in Fig. 1c) and the monolayer $\mathrm{MoSe}_{2}$ (black line in Fig. 1c), respectively (see Supplementary Note 4 ). This simultaneous quenching of PL at both $\mathrm{MoSe}_{2}$ and $\mathrm{WS}_{2} \mathrm{~A}$ excitons was observed in all the heterostructures we constructed, including three $\mathrm{MoSe}_{2} /$ $\mathrm{WS}_{2}$ heterostructures on $\mathrm{SiO}_{2} / \mathrm{Si}$ substrate and seven heterostructures on $\mathrm{LaF}_{3}$ in the absence of the gate voltage (see Supplementary Notes 1 and 7). It thus suggests a type II alignment for the as-prepared $\mathrm{MoSe}_{2} / \mathrm{WS}_{2}$ heterostructures, and the PL quenching is a result of the optically excited electron transferred to the $\mathrm{MoSe}_{2}$ layer and hole transferred to the $\mathrm{WS}_{2}$, according to the band alignment ${ }^{31,32}$ shown schematically in the inset of Fig. 1c. It is interesting to note that the quenching of $\mathrm{MoSe}_{2} \mathrm{~A}$ exciton PL is significantly less than that of the $\mathrm{WS}_{2} \mathrm{~A}$ exciton in the heterojunction region. While the integrated PL of the $\mathrm{WS}_{2} \mathrm{~A}$ exciton in the heterojunction is quenched by more than one order of magnitude smaller, the integrated PL intensity of $\mathrm{MoSe}_{2} \mathrm{~A}$ exciton in the heterojunction is only slightly quenched, being $\sim 70 \%$ of that from the monolayer $\mathrm{MoSe}_{2}$ (Fig. 1c). The significantly less quenching of $\mathrm{MoSe}_{2}$ A exciton PL can be understood from the relative band alignment shown in the inset of Fig. 1c. In the type II alignment configuration, the conduction band minimum (CBM) of the $\mathrm{WS}_{2}$ is only slightly lower than that of the $\mathrm{MoSe}_{2}$ according to the theoretical calculations ${ }^{1,32}$. The thermal equilibrium of the two CBMs at room temperature therefore allows a certain population of electrons in the CBM of the $\mathrm{MoSe}_{2}$ even though the CBM of the $\mathrm{WS}_{2}$ is the lower energy state for electrons in the heterojunction region. The small energy difference between the two CBMs offers the opportunity for us to apply an efficient electrostatic gating to manipulate the optically excited carrier transfer across the $\mathrm{MoSe}_{2} / \mathrm{WS}_{2}$ interface. We achieve that by using the $\mathrm{LaF}_{3}$ as the ionic back gate, which has been proven to efficiently gate $2 \mathrm{D}$ materials though a double layer $^{30}$ (schematically shown in Fig. 1b).

Gate-dependent PL enhancement in $\mathrm{MoSe}_{2} / \mathrm{WS} 2$. To reveal the effect of gate-controlled carrier transfer across the heterojunction, we then investigate the PL spectra around the $\mathrm{MoSe}_{2} \mathrm{~A}$ exciton resonance as a function of the gate voltage for both monolayer $\mathrm{MoSe}_{2}$ (Fig. 2a) and $\mathrm{MoSe}_{2} / \mathrm{WS}_{2}$ heterojunction (Fig. 2b) (see Supplementary Note 5). The CW laser excitation centered at $2.0 \mathrm{eV}(620 \mathrm{~nm})$ with a power of $100 \mu \mathrm{W}$ was used to obtain the PL spectra shown in Fig. 2a, b. This excitation photon energy is

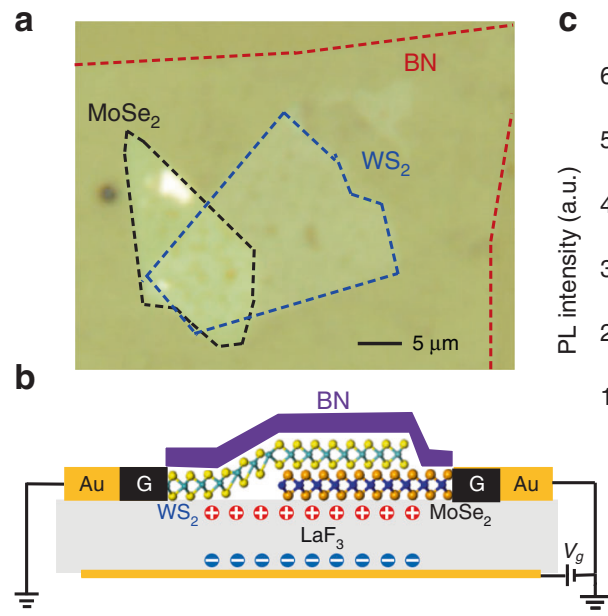

C

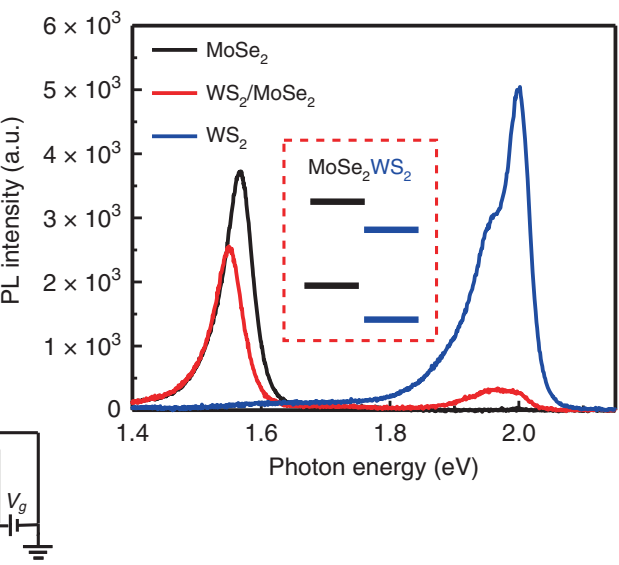

Fig. 1 Monolayer $\mathbf{M o S e}_{2} / \mathbf{W S}_{\mathbf{2}}$ heterostructure device. a Optical microscopic image of the monolayer MoSe $2 / \mathrm{WS}_{2}$ heterostructure, capped with a fewlayer h-BN layer. Scale bar: $5 \mu \mathrm{m}$. b Schematic of the $\mathrm{MoSe}_{2} / \mathrm{WS}_{2}$ heterostructure device, contacted by few-layer graphene electrodes and gated by the ionic substrate $\mathrm{LaF}_{3}$. c Typical room temperature $\mathrm{PL}$ spectra from regions of the monolayer $\mathrm{MoSe}_{2}$ (black), monolayer $\mathrm{WS}_{2}\left(\mathrm{blue}\right.$ ), and $\mathrm{MoSe}_{2} / \mathrm{WS}_{2}$ heterojunction (red), with no gate voltage applied. Inset: schematic representation of the type II band alignment of the $\mathrm{MoSe}_{2} / \mathrm{WS}_{2}$ heterostructure. 
a

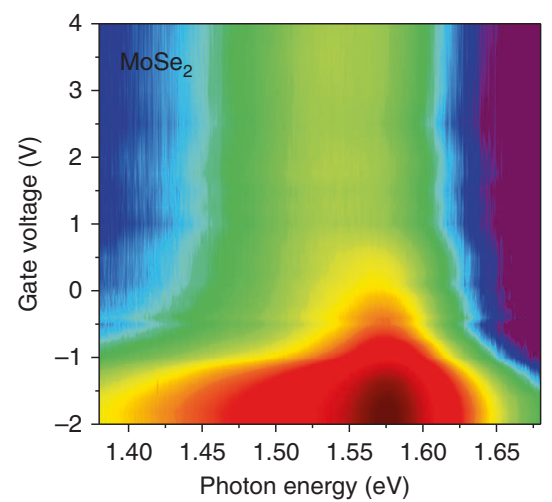

C

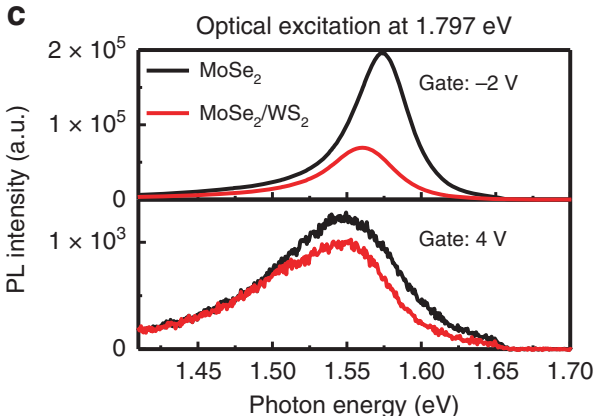

b

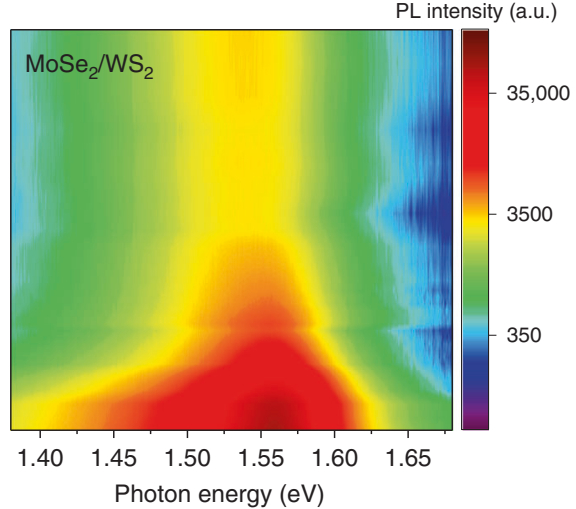

d

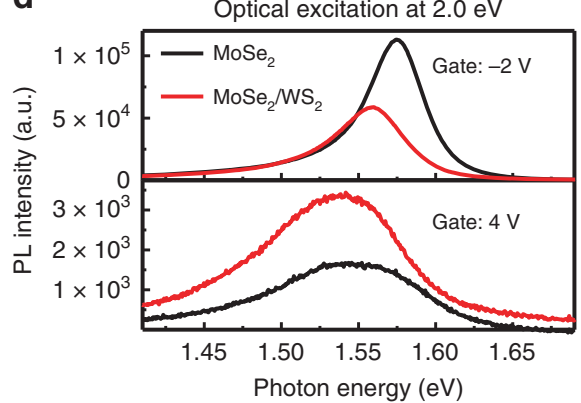

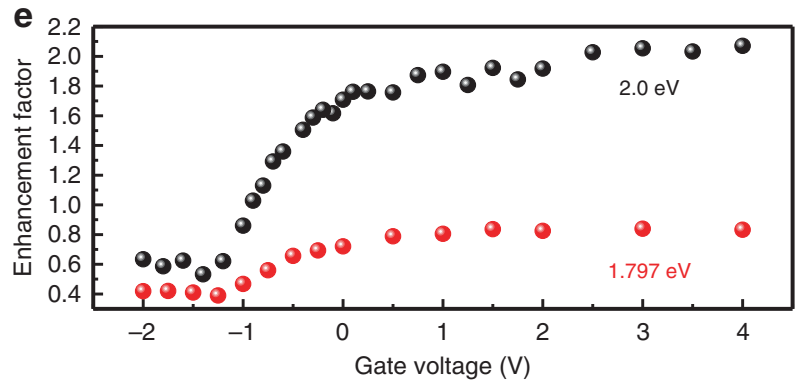

Fig. 2 Gate voltage-tunable transition from PL quenching to PL enhancement in the $\mathbf{M o S e}_{\mathbf{2}} / \mathbf{W S}_{\mathbf{2}}$ heterojunction. a The color plot of the PL spectra for monolayer $\mathrm{MoSe}_{2}$ and $\mathbf{b}$ the color plot of the PL spectra for $\mathrm{MoSe}_{2} / \mathrm{WS}_{2}$ heterostructure region as a function of the gate voltage, under the continuous wave $(\mathrm{CW})$ photoexcitation centered at $2.0 \mathrm{eV}$ and with the excitation power of $100 \mu \mathrm{W}$. The color represents the integrated PL intensity at MoSe $\mathrm{A}_{2}$ exciton resonance. All spectra were taken at room temperature. $\mathbf{c}$, $\mathbf{d}$ are $\mathrm{PL}$ spectra of the monolayer $\mathrm{MoSe}_{2}$ (black) and $\mathrm{MoSe}_{2} / \mathrm{WS}_{2}$ heterojunction (red) under the CW photoexcitation centered at 1.797 and $2.0 \mathrm{eV}$, respectively. The excitation power for both $\mathbf{c}$ and $\mathbf{d}$ is $100 \mu \mathrm{W}$. e The experimentally extracted $\mathrm{PL}$ enhancement factor as a function of the gate voltage for the photoexcitation centered at $2.0 \mathrm{eV}$ (black dots) and $1.797 \mathrm{eV}$ (red dots).

large enough to excite A excitons in both monolayer $\mathrm{MoSe}_{2}$ $(1.548 \mathrm{eV})$ and $\mathrm{WS}_{2}(1.979 \mathrm{eV})$. The PL intensity from the $\mathrm{WS}_{2} \mathrm{~A}$ exciton is drastically quenched in the heterojunction, and we focus on the PL intensity of the $\mathrm{MoSe}_{2} \mathrm{~A}$ exciton for both the monolayer (Fig. 2a) and heterojunction region (Fig. 2b). We can see from the color plots (Fig. 2a, b) that, although the $\mathrm{MoSe}_{2} \mathrm{~A}$ exciton PL intensity is weaker in $\mathrm{MoSe}_{2} / \mathrm{WS}_{2}$ heterojunction (Fig. 2b) than in the monolayer $\mathrm{MoSe}_{2}$ (Fig. 2a) for the gate voltage from $\sim-2$ to $-1 \mathrm{~V}$, the PL is stronger in the heterojunction than in the monolayer $\mathrm{MoSe}_{2}$ at the gate voltage $>0 \mathrm{~V}$. This relative PL ratio from quenching to enhancement transition is clearly illustrated in the PL spectra in Fig. 2d, which combine the line cuts of Fig. $2 \mathrm{a}, \mathrm{b}$ at the gate voltage -2 and $4 \mathrm{~V}$. To better understand the PL behavior change, we define the PL enhancement factor $(\mathrm{EF})$ as $\mathrm{EF}=I_{\mathrm{Heter}} / I_{\mathrm{MoSe}_{2}}$, where $I_{\mathrm{Heter}}\left(I_{\mathrm{MoSe}_{2}}\right)$ is the integrated $\mathrm{MoSe}_{2} \mathrm{~A}$ exciton PL intensity in the $\mathrm{MoSe}_{2} / \mathrm{WS}_{2}$ heterojunction (monolayer $\mathrm{MoSe}_{2}$ ). $\mathrm{EF}$ as a function of the gate voltage for the photoexcitation centered at $2.0 \mathrm{eV}$ is shown in
Fig. 2e (black dots), which shows that $\mathrm{EF}$ is almost a constant between the gate voltage of -2 to $-1 \mathrm{~V}(\mathrm{EF} \sim 0.6)$ but quickly rises to $\sim 1.8$ at the gate voltage $0 \mathrm{~V}$, and it remains largely a constant as the gate voltage is further increased.

It is interesting to note that this observation is sensitive to the excitation photon energy, and the results are distinctively different for the CW photoexcitation of the same power $(100 \mu \mathrm{W})$ but centered at $1.797 \mathrm{eV}(690 \mathrm{~nm})$, which is below the A exciton resonance energy of $\mathrm{WS}_{2}$ but above that of $\mathrm{MoSe}_{2}$. At the gate voltage of $-4 \mathrm{~V}$, we observe PL quenching at $\mathrm{MoSe}_{2} \mathrm{~A}$ exciton resonance in the heterojunction (Fig. 2d), similar to the scenario with the photoexcitation at $2 \mathrm{eV}$ (Fig. 2c). However, as we increase the gate voltage to $4 \mathrm{~V}$, we do not observe the PL enhancement of the $\mathrm{MoSe}_{2} \mathrm{~A}$ exciton in the heterojunction, even though the PL intensity is quite close to that of the monolayer $\mathrm{MoSe}_{2}$ (Fig. 2d). A detailed gate-dependence study of the photoexcitation centered at $1.797 \mathrm{eV}$ also results in quantitative EF as shown in Fig. 2e (red dots), which shows a similar step function behavior as the case of 

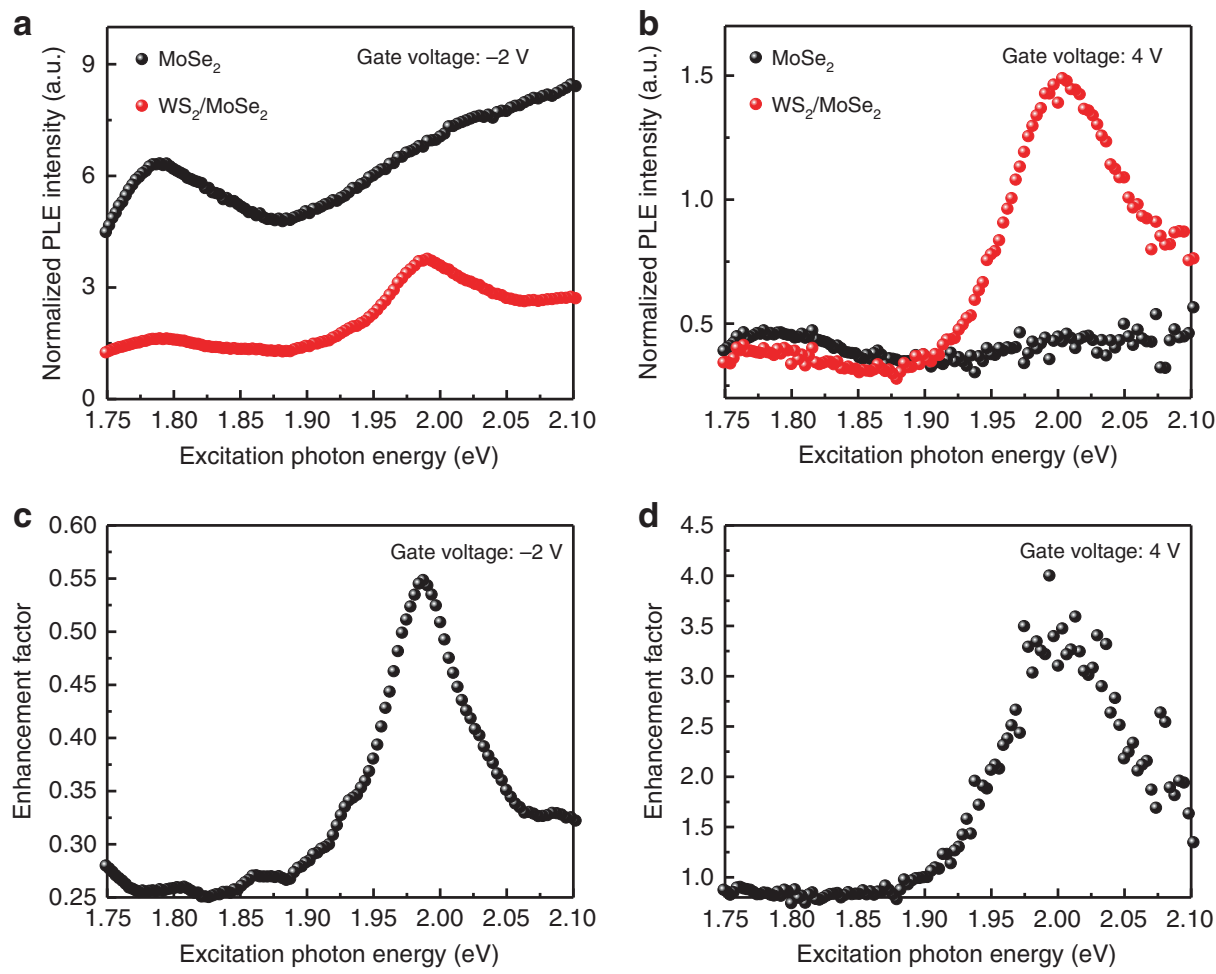

Fig. 3 PLE spectra of the monolayer $\mathbf{M o S e}_{\mathbf{2}}$ and $\mathbf{M o S e}_{\mathbf{2}} / \mathbf{W S}_{\mathbf{2}}$ heterostructure for different gate voltages. $\mathbf{a}, \mathbf{b}$ are integrated PL intensity at MoSe $e_{2} \mathrm{~A}$ exciton resonance as a function of the excitation photon energy for monolayer $\mathrm{MoSe}_{2}$ (black) and $\mathrm{MoSe}_{2} / \mathrm{WS}_{2}$ heterojunction (red) regions at the gate voltage of $-2 \mathrm{~V}(\mathbf{a})$ and the gate voltage of $4 \mathrm{~V}(\mathbf{b})$, respectively. $\mathbf{c}$, $\mathbf{d}$ are PL enhanced factor for the gate voltage of $-2 \mathrm{~V}(\mathbf{c})$ and $4 \mathrm{~V}(\mathbf{d})$, respectively.

photoexcitation centered at $2.0 \mathrm{eV}$, but the maximum value of $\mathrm{EF}$ is smaller and never exceeds 1 .

PL excitation (PLE) spectroscopy of EF in $\mathrm{MoSe}_{2} / \mathrm{WS}_{2}$. Since the observed PL EF at $\mathrm{MoSe}_{2} \mathrm{~A}$ exciton resonance is sensitive to the excitation photon energy, we then perform a detailed PLE spectroscopic study. The integrated PL intensity at the $\mathrm{MoSe}_{2} \mathrm{~A}$ exciton resonance for monolayer $\mathrm{MoSe}_{2}$ (black) and $\mathrm{MoSe}_{2} / \mathrm{WS}_{2}$ heterojunction (red) are plotted as a function of the excitation photon energy in Fig. 3a, b for the gate voltage of -2 and $4 \mathrm{~V}$, respectively. Figure $3 \mathrm{c}$ shows the $\mathrm{EF}$ for the gate voltage of $-2 \mathrm{~V}$, and when both $\mathrm{MoSe}_{2}$ and $\mathrm{WS}_{2}$ are intrinsic, the $\mathrm{MoSe}_{2} \mathrm{~A}$ exciton $\mathrm{PL}$ intensity is always quenched for different excitation photon energies, with the peak value of 0.55 at the excitation photon energy of $\sim 2.0 \mathrm{eV}$. However, at the gate voltage of $4 \mathrm{~V}$, the $\mathrm{EF}$ is largely flat and slightly $<1 \quad(\sim 0.8)$ when the excitation photon energy is $<1.9 \mathrm{eV}$. When the excitation photon energy $>1.9 \mathrm{eV}$, the PL enhancement effect starts to occur with the EF $>1$. The EF reaches the maximum value when the excitation photon energy is about $2.0 \mathrm{eV}$. The excitation photon energy for the peaked EF value in Fig. $3 c$, d coincides with the $\mathrm{A}$ exciton resonance of monolayer $\mathrm{WS}_{2}$, which suggests that photoexcited carrier transfer from $\mathrm{WS}_{2}$ to $\mathrm{MoSe}_{2}$ plays the central role in the PL enhancement in the $\mathrm{MoSe}_{2} / \mathrm{WS}_{2}$ heterojunction, which, as a result, explains the lack of the PL enhancement with the off-resonance excitation at $1.797 \mathrm{eV}$ (Fig. 2d).

It is worth noting that, even with the CW photoexcitation centered at $2.0 \mathrm{eV}$, we have not observed that the PL EF exceeds 1 for three $\mathrm{MoSe}_{2} / \mathrm{WS}_{2}$ heterojunction devices fabricated on $\mathrm{SiO}_{2} / \mathrm{Si}$ substrate (300-nm-thick thermal oxide), with the silicon back gate voltage as high as $80 \mathrm{~V}$ (see Supplementary Note 1). This observation suggests that the efficient gating from $\mathrm{LaF}_{3}$ is essential for realizing the PL enhancement in the heterojunction.
Previous work has shown that the $\mathrm{LaF}_{3}$ back gate should be at least $>100$ times more efficient than the silicon back gate with $300 \mathrm{~nm}$ thermal oxide ${ }^{30}$.

\section{Discussion}

The experimental observation can be understood theoretically by considering the gate-dependent carrier distribution in the heterostructure. Taking into account the quantum capacitance of the monolayer $\mathrm{MoSe}_{2}$ and $\mathrm{WS}_{2}$, for the device configuration shown in Fig. $1 b$, the effective capacitance model can be schematically shown as the inset of Fig. $4\left(\mathrm{MoSe}_{2}\right.$ being the bottom layer, and detailed derivation in Supplementary Note 3). Here $C_{Q 1}\left(C_{Q 2}\right)$ are the quantum capacitance of monolayer $\mathrm{MoSe}_{2}\left(\mathrm{WS}_{2}\right), C_{G 1}$ is the geometry capacitance between $\mathrm{MoSe}_{2}$ and the $\mathrm{LaF}_{3}$ back gate, and $C_{G 2}$ is the geometry capacitance between $\mathrm{MoSe}_{2}$ and $\mathrm{WS}_{2}$. For qualitative understanding, we consider zero-temperature case here (see Supplementary Note 2 for the discussion of the finite temperature case, which does not qualitatively change the picture). Owing to the large energy difference between the VBMs of $\mathrm{MoSe}_{2}$ and $\mathrm{WS}_{2}$, the hole transfer from $\mathrm{WS}_{2}$ to $\mathrm{MoSe}_{2}$ (when $\mathrm{WS}_{2}$ is optically excited) is always $\sim 100 \%$. As a result, we focus on the gate dependence of the electron transfer from $\mathrm{MoSe}_{2}$ to $\mathrm{WS}_{2}$. As shown in Fig. 4, when the gate voltage is at point A (e.g., $-2 \mathrm{~V}$ for the device 2 shown in Fig. 2), both the $\mathrm{MoSe}_{2}$ and $\mathrm{WS}_{2}$ layers are intrinsic and with the quantum capacitance of zero. As a result, the gate voltage is dropped only on the quantum capacitance and the band alignment is determined by the work function of each layer. The type II alignment (shown at point A in Fig. 4) determines that the optically excited electron in $\mathrm{MoSe}_{2}$ will transfer to $\mathrm{WS}_{2}$, reducing the electron density in the $\mathrm{MoSe}_{2}$ layer in the heterojunction, compared to the case of the bare monolayer $\mathrm{MoSe}_{2}$. In addition, with (on-resonance excitation) and without (off-resonance excitation) the hole transfer from $\mathrm{WS}_{2}$ to $\mathrm{MoSe}_{2}$, 


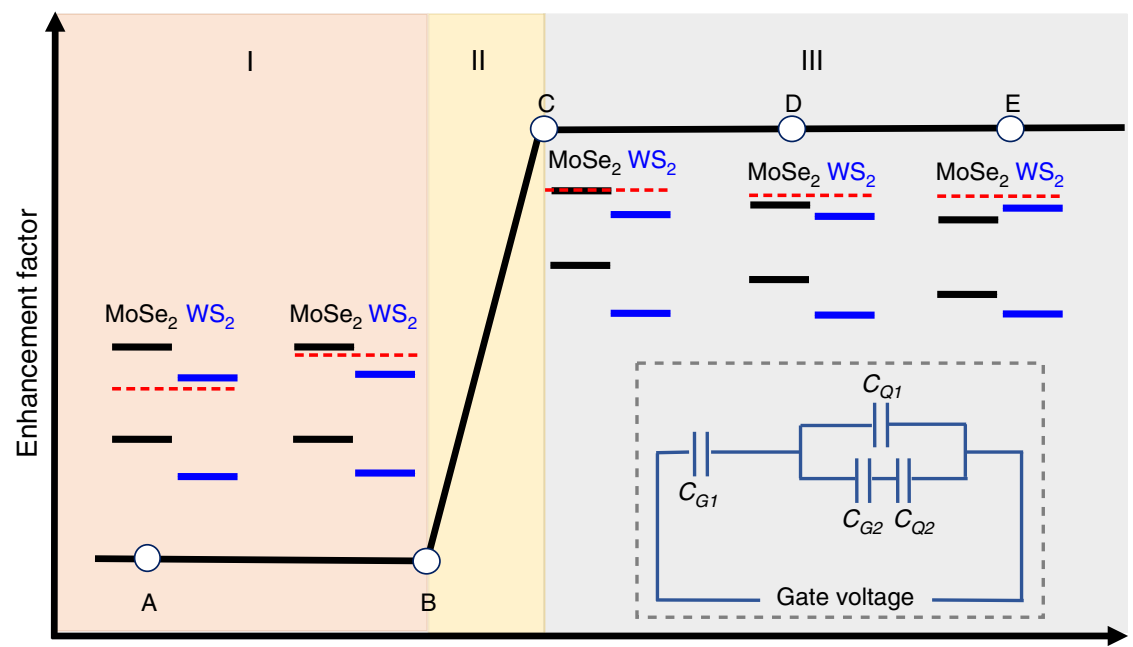

Gate voltage (V)

Fig. 4 Theoretical understanding of the PL quenching to PL enhancement transition. The enhancement factor as a function of the gate voltage clearly exhibits three distinct regions. Schematics of band alignment of the $\mathrm{MoSe}_{2} / \mathrm{WS}_{2}$ heterojunction, along with the Fermi energy level (dashed line), are labeled for different points to explain the different PL enhancement factor behaviors. Inset: schematic of the effective capacitance circuit of the MoSe $e_{2}$ $W_{2}$ heterojunction with the device configuration shown in Fig. $1 \mathrm{~b}$.

the electron density in the $\mathrm{MoSe}_{2}$ layer in the heterojunction is always less than the hole density. As a result, the electron is the minor carrier that determines the available $\mathrm{MoSe}_{2} \mathrm{~A}$ exciton density. The reduced electron density thus leads to the quenching of $\mathrm{MoSe}_{2} \mathrm{~A}$ exciton PL in the heterojunction.

When the $\mathrm{WS}_{2}$ layer starts to get electron-doped (point $\mathrm{B}$ ), the number of optically excited electrons transferred from the $\mathrm{MoSe}_{2}$ to $\mathrm{WS}_{2}$ in the heterojunction region will be modulated by the gate voltage. For simplicity, we can use the off-resonance excitation as an example. The charge transfer from $\mathrm{MoSe}_{2}$ to $\mathrm{WS}_{2}, \Delta Q$, can be obtained from the following equation according to the effective capacitance model $^{33}$ (inset of Fig. 4):

$$
\frac{Q-\Delta Q}{C_{Q 1}}=\frac{\Delta Q}{C_{Q 2}}+\frac{\Delta Q}{C_{G 2}},
$$

where $Q$ is the total charge of optically excited electrons in the $\mathrm{MoSe}_{2}$ layer of the $\mathrm{MoSe}_{2} / \mathrm{WS}_{2}$ heterojunction. Reorganization of Eq. (1) results in the expression of $\Delta Q$ as:

$$
\frac{\Delta Q}{Q}=\frac{1}{1+\frac{C_{Q 1}}{C_{Q 2}}+\frac{C_{Q 1}}{C_{G 2}}} .
$$

For gate voltage smaller than that of point $\mathrm{B}, C_{Q 1}=0$ and hence $\Delta Q=Q$, which indicates that $\sim 100 \%$ of the optically excited electron in $\mathrm{MoSe}_{2}$ layer of the heterojunction region is transferred to $\mathrm{WS}_{2}$. As a result, PL quenching of the $\mathrm{MoSe}_{2}$ layer in the heterojunction is similar to that of A point (similar EF). As we move forward from point $\mathrm{B}$, however, electron transfer will be less efficient due to the finite $C_{Q 1}$ (i.e., finite density of states (DOS) at Fermi level in $\mathrm{MoSe}_{2}$ ) and the PL quenching will be less significant. As the gate voltage is increased to point $\mathrm{C}$, the doping further increases and the Fermi level is aligned with the conduction band of $\mathrm{MoSe}_{2}$. Assuming a similar effective electron mass $m$ in $\mathrm{WS}_{2}$ and $\mathrm{MoSe}_{2}$, we have $C_{\mathrm{Q} 1}=C_{\mathrm{Q} 2}=C_{\mathrm{Q}}$, where $C_{\mathrm{Q}}=\frac{m}{\pi \hbar^{2}}$ is the DOS in $2 \mathrm{D}$. Since $C_{Q} \gg C_{G 2}$ (see Supplementary Note 2), from Eq. (2), we found that $\Delta Q \sim 0$ and optically excited electron transfer from $\mathrm{MoSe}_{2}$ to $\mathrm{WS}_{2}$ is blocked. The EF of $\mathrm{MoSe}_{2} \mathrm{~A}$ exciton PL will therefore again be largely a constant, with the value of 1 (Fig. 4) for the off-resonance excitation in the ideal scenario.
The electron transfer in the on-resonance scenario can be understood in a similar fashion (see Supplementary Note 2), with similar PL quenching $(\mathrm{EF}<1)$ from point $\mathrm{A}$ to $\mathrm{B}$. However, when $\mathrm{MoSe}_{2}$ is sufficiently doped (point C), optically induced holes in the $\mathrm{MoSe}_{2}$ layer become the minor carrier that determines the $\mathrm{MoSe}_{2} \mathrm{~A}$ exciton density. For the on-resonance excitation, the $\mathrm{WS}_{2}$ layer is also excited and we have nearly $100 \%$ of the optically excited holes transfer from $\mathrm{WS}_{2}$ to $\mathrm{MoSe}_{2}$. Therefore, the A exciton density in the $\mathrm{MoSe}_{2}$ layer in the heterojunction is enhanced, giving rise to the PL enhancement with a largely constant $\mathrm{EF}>1$. We thus conclude that, for both the offresonance and on-resonance excitation, the qualitative gate dependence of EF will be of the form shown in Fig. 4. Particularly, EF will show an abrupt increase around specific gate voltage (region II) and remain largely constant on either side. On the low voltage side (region I), EF should be $<1$; and on the high gate voltage side (region III), $\mathrm{EF}=1$ for the off-resonance excitation and $\mathrm{EF}>1$ for the on-resonance excitation.

The theoretical prediction in Fig. 4 is in excellent agreement with our experimental observation in Fig. 2e. The experimentally observed EF as a function of the gate voltage can be clearly divided into three regions, similar to a step function for both the on-resonance and off-resonance excitation as predicted by Fig. 4 . The EF for the on-resonance excitation (photoexcitation at 2.0 $\mathrm{eV}$ ) in region III shows an $\mathrm{EF} \sim 2.0$, while the EF for the offresonance excitation (photoexcitation at $1.797 \mathrm{eV}$ ) in the region III is about 0.8 . The EF of $<1$ for the off-resonance case is probably due to decreased quantum efficiency in the heterojunction from the different dielectric environment.

The consideration of the finite temperature effects is included in Supplementary Note 2, and it gives qualitatively similar results as in Fig. 4. Interestingly, we found that, for large enough gate voltage, the charge accumulated on the $C_{G 2}$ will give rise to a large energy shift between the $\mathrm{MoSe}_{2}$ and $\mathrm{WS}_{2}$, which switches the type II alignment to a type I alignment configuration, as shown schematically by the inset at point $\mathrm{E}$ in Fig. 4 . The efficient ionic gating thus not only allows the control of optically excited carrier transfer across the atomically sharp interface but also leads to the possibility of modifying the alignment type reversibly. The associated fundamental understanding will enable quantum 
optoelectronics based on transition metal dichalcogenide (TMDC) vdW heterostructures.

\section{Method}

Device fabrication. The $\mathrm{MoSe}_{2} / \mathrm{WS}_{2}$ heterostructure devices were fabricated through a layer-by-layer dry transfer technique ${ }^{29}$. More specifically, each of the monolayer TMDC was sequentially transferred to the $\mathrm{LaF}_{3}$ substrate, and a final $\mathrm{BN}$ flake was used to cap the heterostructure. Two pieces of few-layer graphene were used as the electrodes to contact the monolayer $\mathrm{MoSe}_{2}$ and $\mathrm{WS}_{2}$ layer separately, and both were grounded during the measurements, as schematically shown in Fig. 1b. The final devices were annealed in vacuum at $100^{\circ} \mathrm{C}$ for $3 \mathrm{~h}$.

Optical measurements. All the optical measurements in this work were performed at room temperature. The micro-PL measurements were performed with a homebuilt confocal microscope, in which the excitation lasers were focused to a spot size of $\sim 2 \mu \mathrm{m}$. The PLE spectra were taken with a supercontinuum white laser (Fianium), and the filtered light (with bandwidth $\sim 4 \mathrm{~nm}$ ) was used as the excitation source.

\section{Data availability}

The data that support the findings of this study are available from the authors on reasonable request, see "Author contributions" for specific data sets.

Received: 6 January 2020; Accepted: 24 April 2020;

Published online: 26 May 2020

\section{References}

1. Paik, E. Y. et al. Interlayer exciton laser of extended spatial coherence in atomically thin heterostructures. Nature 576, 80-84 (2019).

2. Zhang, K. et al. Interlayer transition and infrared photodetection in atomically thin type-II $\mathrm{MoTe}_{2} / \mathrm{MoS}_{2}$ van der Waals heterostructures. ACS Nano 10, 3852-3858 (2016).

3. Zhang, W. et al. Ultrahigh-gain photodetectors based on atomically thin graphene- $\mathrm{MoS}_{2}$ heterostructures. Sci. Rep. 4, 3826 (2014)

4. Zhou, X. et al. Vertical heterostructures based on $\mathrm{SnSe}_{2} / \mathrm{MoS}_{2}$ for high performance photodetectors. 2D Mater. 4, 25048 (2017).

5. Huang, M. et al. Broadband black-phosphorus photodetectors with high responsivity. Adv. Mater. 28, 3481-3485 (2016).

6. Splendiani, A. et al. Emerging photoluminescence in monolayer $\mathrm{MoS}_{2}$. Nano Lett. 10, 1271-1275 (2010)

7. Mak, K. F., Lee, C., Hone, J., Shan, J. \& Heinz, T. F. Atomically thin MoS 2: a new direct-gap semiconductor. Phys. Rev. Lett. 105, 136805 (2010).

8. Mak, K. F. et al. Tightly bound trions in monolayer $\mathrm{MoS}_{2}$. Nat. Mater. 12, 207-211 (2012).

9. Ross, J. S. et al. Electrical control of neutral and charged excitons in a monolayer semiconductor. Nat. Commun. 4, 1474 (2013).

10. Zhu, B., Chen, X. \& Cui, X. Exciton binding energy of monolayer $\mathrm{WS}_{2} . S c i$ Rep. 5, 9218 (2015).

11. Gerd, P. et al. Identification of excitons, trions and biexcitons in single-layer $\mathrm{WS}_{2}$. Phys. Status Solidi Rapid Res. Lett. 9, 457-461 (2015).

12. Singh, A. et al. Coherent electronic coupling in atomically thin $\mathrm{MoS}_{2}$. Phys Rev. Lett. 112, 216804 (2014).

13. Li, Z. et al. Revealing the biexciton and trion-exciton complexes in BN encapsulated $\mathrm{WSe}_{2}$. Nat. Commun. 9, 3719 (2018).

14. Rivera, P. et al. Interlayer valley excitons in heterobilayers of transition metal dichalcogenides. Nat. Nanotechnol. 13, 1004-1015 (2018).

15. Jin, C. et al. Ultrafast dynamics in van der Waals heterostructures. Nat. Nanotechnol. 13, 994-1003 (2018).

16. Yu, Y. et al. Equally efficient interlayer exciton relaxation and improved absorption in epitaxial and nonepitaxial $\mathrm{MoS}_{2} / \mathrm{WS}_{2}$ heterostructures. Nano Lett. 15, 486-491 (2015).

17. Rigosi, A. F., Hill, H. M., Li, Y., Chernikov, A. \& Heinz, T. F. Probing interlayer interactions in transition metal dichalcogenide heterostructures by optical spectroscopy: $\mathrm{MoS}_{2} / \mathrm{WS}_{2}$ and $\mathrm{MoSe}_{2} / \mathrm{WSe}_{2}$. Nano Lett. 15, 5033-5038 (2015).

18. Lee, C.-H. et al. Atomically thin $\mathrm{p}-\mathrm{n}$ junctions with van der Waals heterointerfaces. Nat. Nanotechnol. 9, 676-681 (2014).

19. Kim, J. et al. Observation of ultralong valley lifetime in $\mathrm{WSe}_{2} / \mathrm{MoS}_{2}$ heterostructures. Sci. Adv. 3, e1700518 (2017).

20. Zhu, X. et al. Charge transfer excitons at van der Waals interfaces. J. Am. Chem. Soc. 137, 8313-8320 (2015).

21. Hong, X. et al. Ultrafast charge transfer in atomically thin $\mathrm{MoS}_{2} / \mathrm{WS}_{2}$ heterostructures. Nat. Nanotechnol. 9, 682 (2014).

22. Bellus, M. Z. et al. Type-I van der Waals heterostructure formed by $\mathrm{MoS}_{2}$ and $\mathrm{ReS}_{2}$ monolayers. Nanoscale Horiz. 2, 31-36 (2017).
23. Baranowski, M. et al. Probing the interlayer exciton physics in a $\mathrm{MoS}_{2} /$ $\mathrm{MoSe}_{2} / \mathrm{MoS}_{2}$ van der Waals heterostructure. Nano Lett. 17, 6360-6365 (2017).

24. Nagler, P. et al. Interlayer exciton dynamics in a dichalcogenide monolayer heterostructure. 2D Mater. 4, 25112 (2017).

25. Ross, J. S. et al. Interlayer exciton optoelectronics in a $2 \mathrm{D}$ heterostructure $\mathrm{p}-\mathrm{n}$ junction. Nano Lett. 17, 638-643 (2017).

26. Miller, B. et al. Long-lived direct and indirect interlayer excitons in van der Waals heterostructures. Nano Lett. 17, 5229-5237 (2017).

27. Rivera, P. et al. Observation of long-lived interlayer excitons in monolayer $\mathrm{MoSe}_{2}-\mathrm{WSe}_{2}$ heterostructures. Nat. Commun. 6, 6242 (2015).

28. Kozawa, D. et al. Evidence for fast interlayer energy transfer in $\mathrm{MoSe}_{2} / \mathrm{WS}_{2}$ heterostructures. Nano Lett. 16, 4087-4093 (2016).

29. Li, Z. et al. Emerging photoluminescence from the dark-exciton phonon replica in monolayer WSe 2 . Nat. Commun. 10, 2469 (2019).

30. Wu, C.-L. et al. Gate induced metal-insulator transition in $\mathrm{MoS}_{2}$ by solid superionic conductor $\mathrm{LaF}_{3}$. Nano Lett. 18, 2387-2392 (2018).

31. Alexeev, E. M. et al. Resonantly hybridized excitons in moiré superlattices in van der Waals heterostructures. Nature 567, 81-86 (2019).

32. Ruiz-Tijerina, D. A. \& Fal'ko, V. I. Interlayer hybridization and moiré superlattice minibands for electrons and excitons in heterobilayers of transition-metal dichalcogenides. Phys. Rev. B 99, 125424 (2019).

33. Luryi, S. Quantum capacitance devices. Appl. Phys. Lett. 52, 501-503 (1988).

\section{Acknowledgements}

T.W. and S.-F.S. acknowledge support from ACS PRF through grant 59957-DNI10. S.M., Z.Li, and S.-F.S. acknowledge support from AFOSR through Grant FA9550-18-1-0312. Z.Lian and S.-F.S. acknowledge support from NYSTAR through Focus Center-NY-RPI Contract C150117. The device fabrication was supported by the Micro and Nanofabrication Clean Room (MNCR) at Rensselaer Polytechnic Institute (RPI). K.W. and T.T. acknowledge support from the Elemental Strategy Initiative conducted by the MEXT, Japan and the CREST (JPMJCR15F3), JST. F.S. acknowledges support from the National Natural Science Foundation of China (No. U1732273). S.-F.S. also acknowledges the support from a KIP grant from RPI and a VSP grant from NHMFL.

\section{Author contributions}

S.-F.S. conceived the experiment. Y.M. and Z.Lian fabricated the devices. Y.M. and T.W. performed the measurements. S.-F.S., T.W., Y.M., C.J., S.M., Z.Li and F.S. analyzed the data. T.T. and K.W. grew the BN crystals. S.-F.S. supervised the project. S.-F.S., T.W., C.J., S.M., and Y.M. wrote the manuscript with input from all the other co-authors. All authors discussed the results and contributed to the manuscript.

\section{Competing interests}

The authors declare no competing interests.

\section{Additional information}

Supplementary information is available for this paper at https://doi.org/10.1038/s41467020-16419-x.

Correspondence and requests for materials should be addressed to F.S. or S.-F.S.

Peer review information Nature Communications thanks the anonymous reviewers for their contribution to the peer review of this work. Peer review reports are available.

Reprints and permission information is available at http://www.nature.com/reprints

Publisher's note Springer Nature remains neutral with regard to jurisdictional claims in published maps and institutional affiliations.

Open Access This article is licensed under a Creative Commons Attribution 4.0 International License, which permits use, sharing, adaptation, distribution and reproduction in any medium or format, as long as you give appropriate credit to the original author(s) and the source, provide a link to the Creative Commons license, and indicate if changes were made. The images or other third party material in this article are included in the article's Creative Commons license, unless indicated otherwise in a credit line to the material. If material is not included in the article's Creative Commons license and your intended use is not permitted by statutory regulation or exceeds the permitted use, you will need to obtain permission directly from the copyright holder. To view a copy of this license, visit http://creativecommons.org/ licenses/by/4.0/.

(c) The Author(s) 2020 